\title{
Szenario-Technik mit digitalen Technologien
}

\author{
Patrick Ködding ${ }^{1(\bowtie)}$ und Roman Dumitrescu ${ }^{1,2}$ \\ ${ }^{1}$ Heinz Nixdorf Institut, Universität Paderborn, 33102 Paderborn, Deutschland \\ \{Patrick.Koedding, Roman. Dumitrescu\} @hni.upb.de \\ ${ }^{2}$ Fraunhofer Institut für Entwurfstechnik Mechatronik, 33102 Paderborn, \\ Deutschland
}

\begin{abstract}
Zusammenfassung. Im Zuge der voranschreitenden Digitalisierung ist das Umfeld von Industrieunternehmen zunehmend durch Volatilität, Unsicherheit, Komplexität und Ambiguität geprägt. Immens ansteigende Datenmengen, die sinkende Halbwertszeit von Informationen und immer kürzere Entwicklungszeiten setzen Unternehmen unter enormen Zeitdruck. Folglich konzentrieren sich Unternehmen verstärkt auf das Tagesgeschäft oder greifen auf Ergebnisse für die strategische Planung zurück, die mit höherem Fokus auf Geschwindigkeit als auf Gründlichkeit erarbeitet wurden. Um langfristig wettbewerbsfähig zu bleiben, müssen sich Unternehmen jedoch systematisch mit zukünftigen Entwicklungen auseinandersetzen. Die Szenario-Technik ist dafür das geeignete Werkzeug. Mithilfe von digitalen Technologien, wie z. B. Anwendungen der Künstlichen Intelligenz (KI), lassen sich die Anwendungsfreundlichkeit erhöhen und der Datenerhebungsaufwand reduzieren.

Dies ist Gegenstand des vorliegenden Beitrags. Zunächst wird die Problematik detaillierter beschrieben. Es folgt die Einordnung der SzenarioTechnik in die Szenario-basierte Vorausschau sowie die Herleitung und Vorstellung ihrer konstituierenden und optionalen Phasen und Schritte. Im Anschluss werden Herausforderungen beim Einsatz der Szenario-Technik beleuchtet. Darauf aufbauend werden Potenziale für einen Erfolg versprechenden Einsatz von digitalen Technologien in der Szenario-Technik aufgezeigt. Abschließend werden anhand der Herausforderungen und Potenziale drei Handlungsfelder für eine Szenario-Technik mit digitalen Technologien abgeleitet: die Integration von digitalen Technologien, die methodische Anpassung und Weiterentwicklung der Szenario-Technik sowie die digitale Souveränität.
\end{abstract}

Schlüsselwörter: Vorausschau $\cdot$ Szenario-Technik · Digitale Technologien · Künstliche Intelligenz · Digitale Souveränität

\section{Einführung}

Die US-amerikanische Managementberatung Bain \& Company hat im Zeitraum von 1993 bis 2017 16-mal weltweit zahlreiche Manager - im Jahr 2017 waren es 1268 zu den wichtigsten „Management Tools \& Trends“ befragt, u. a. auch zur Nutzung von Werkzeugen zur Szenario-basierten Vorausschau. Es zeigt sich, dass Unternehmen solche Werkzeuge verstärkt in Zeiten großer Unsicherheit einsetzen, wie z. B. 
nach dem Platzen der Dotcom-Blase und den Terroranschlägen vom 11. September 2001 oder der Finanzkrise ab 2007. Dies hat zwei Gründe: Einerseits soll mithilfe der Werkzeuge Orientierungswissen in unsicheren, volatilen Umfeldern generiert werden. Andererseits wird durch ihren Einsatz auch die Möglichkeit zur Steigerung des Unternehmenswachstums gesehen. Während in Krisenzeiten die Nutzung von Werkzeugen zur Szenario-basierten Vorausschau (vgl. Abschn. 2) bei teils über $60 \%$ der befragten Unternehmen gelegen hat, ist die Zahl der Nutzung bis 2017 auf etwas mehr als $20 \%$ zurückgegangen (vgl. Abb. 1). Gleichzeitig ist jedoch die Zufriedenheit mit diesen Werkzeugen und ihren erzielten Ergebnissen konstant auf einem hohen Niveau geblieben (Rigby und Bilodeau 2018; Bain \& Company, Inc. 2018).

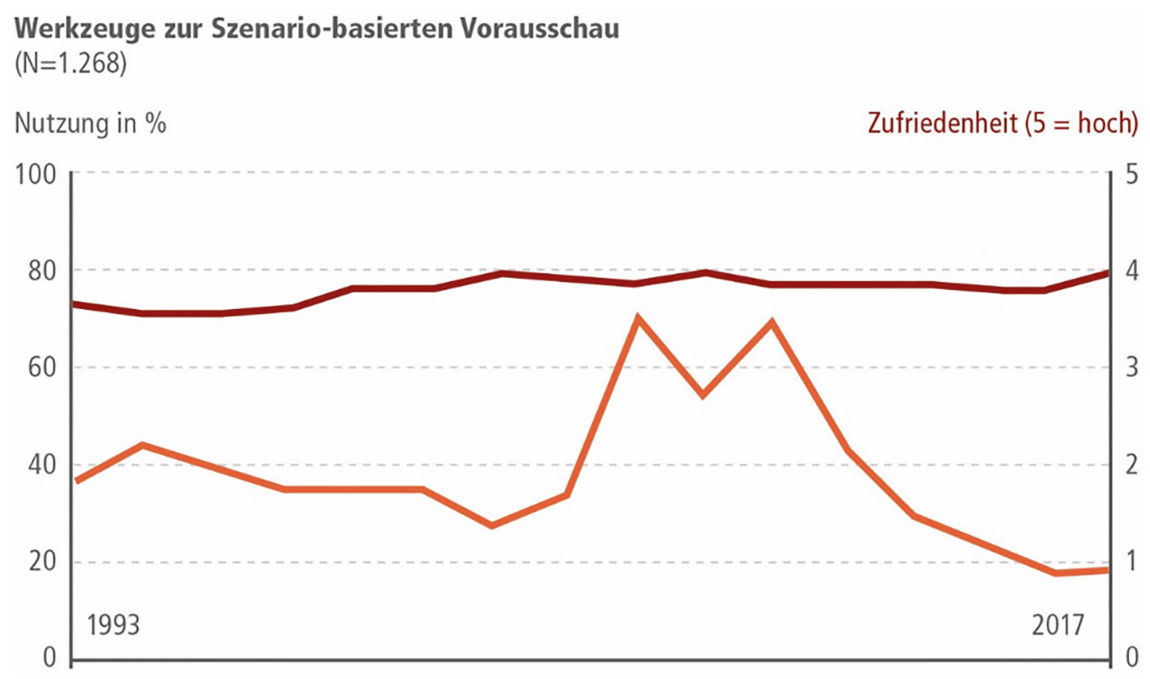

Abb.1. Nutzung und Zufriedenheit beim Einsatz der Szenario-Technik nach. (Eigene Darstellung nach Rigby und Bilodeau 2018; Bain \& Company, Inc. 2018)

Einen wesentlichen Grund für den Rückgang der Nutzungszahlen leitet das McKinsey Global Institute aus einer 15-jährigen Längsschnittstudie mit 615 mittleren und großen US-amerikanischen Unternehmen ab. Im Zuge der Digitalisierung und einem damit verbundenen immer dynamischeren und vernetzten Unternehmensumfeld sehen sich Unternehmen zu verstärkt kurzfristigen Entscheidungen an Stelle von strategischer Planung gezwungen. Die traditionell hohe Komplexität von Methoden der Szenario-basierten Vorausschau erschwert deren Anwendung zusätzlich (Barton et al. 2018).

Unternehmen, die systematisch strategische Vorausschau betreiben, sind langfristig betrachtet jedoch signifikant erfolgreicher und weniger volatil als kurzfristig orientierte Unternehmen. Dies belegen sowohl Zahlen der Studie des McKinsey Global Institute als auch die Ergebnisse des Corporate Foresight Benchmarking Reports, einer Längsschnittstudie mit 83 europäischen Unternehmen. So generieren strategisch planende Unternehmen im Schnitt deutlich höhere Umsätze, erzielen 
deutlich höhere Gewinne und sind deutlich rentabler als ihre Konkurrenten. Darüber hinaus verfügen sie über ein deutlich höheres Wachstum der Marktkapitalisierung als ihre Wettbewerber (Rohrbeck und Kum 2018; Rohrbeck et al. 2018; Barton et al. 2018).

Die Bedeutung der strategischen Vorausschau für Unternehmen lässt sich anhand des sogenannten Vier-Ebenen-Modells der zukunftsorientierten Unternehmensgestaltung verdeutlichen. Auf der ersten Ebene, der Vorausschau, beschäftigen sich Unternehmen systematisch mit denkbaren Entwicklungen von Märkten, Branchen, Technologien etc., um zukünftige Erfolgspotenziale, aber auch Gefahren für das etablierte Geschäft von heute frühzeitig abzuleiten (Gausemeier und Plass 2014). Aufgrund ihrer Beliebtheit in der Praxis und methodischer Vorteile eignen sich hierfür insbesondere Szenario-basierte Methoden der Vorausschau, wie z. B. die SzenarioTechnik(vgl. u. a. Abschn. 2), (Schühly et al. 2020; Ogilvy 2015)

Aufbauend auf der Vorausschau werden Strategien (zweite Ebene) entwickelt, die beschreiben, wie Unternehmen in der zukünftigen Wettbewerbsarena eine vorteilhafte Positionierung erreichen wollen und welche Marktleistungen mit welchen zugehörigen Geschäftsmodellen angeboten werden sollen. Im Zuge der Digitalisierung sind dies verstärkt attraktive digitale Geschäftsmodelle. Auf der dritten Ebene gilt es, die Geschäftsprozesse zur Operationalisierung der Strategie festzulegen, bevor abschließend IT-Systeme (vierte Ebene) zur Unterstützung der wohlstrukturierten Geschäftsprozesse definiert werden (Gausemeier und Plass 2014; Dumitrescu und Gausemeier 2018; Echterhoff et al. 2016).

Folglich stellt sich die Frage, wie Unternehmen dazu befähigt werden können, in einem immer komplexer werdenden Unternehmensumfeld weiterhin bzw. wieder verstärkt Methoden der Szenario-basierten Vorausschau für die zukunftsorientierte Unternehmensgestaltung einzusetzen. Digitale Technologien, zu denen auch Anwendungen auf Basis Künstlicher Intelligenz (KI) zählen, stellen eine Erfolg versprechende Möglichkeit dar, diese Herausforderungen zu adressieren (Schühly et al. 2020). In diesem Beitrag werden erste Handlungsfelder für eine digital souveräne Szenariobasierte Vorausschau mit digitalen Technologien hergeleitet und beschrieben.

\section{Szenario-basierte Vorausschau}

Vorausschau ist ein partizipativer, antizipativer und visionsbildender Prozess mit dem Ziel, einen Blick in die Zukunft zu werfen, um heutige Entscheidungsprozesse zu unterstützen (Becker 2002). Ein Szenario beschreibt in diesem Zusammenhang eine allgemeinverständliche und nachvollziehbare Beschreibung einer möglichen Situation in der Zukunft sowie die Darstellung einer Entwicklung, die aus der Gegenwart zu dieser Situation führen könnte (Gausemeier et al. 2019).

Das Vorausdenken der Zukunft mithilfe von Szenarien basiert dabei auf mehreren Grundprinzipien: 1) Die Zukunft wird in komplexen Bildern beschrieben. Es reicht nicht mehr aus, den Untersuchungsgegenstand (z. B. Werkzeugmaschinenbau) durch einige voneinander unabhängige Einflussfaktoren $\mathrm{zu}$ beschreiben. In der Regel geht es um komplexe Systeme, die ein vernetztes Denken erfordern. 2) Es gibt mehrere 
Möglichkeiten, wie sich die Zukunft entwickeln könnte. Damit wird der Erkenntnis Rechnung getragen, dass die Zukunft nicht exakt prognostizierbar ist. Dies wird als multiple Zukunft bezeichnet (Gausemeier et al. 2019). 3) Die Veränderungstreiber der Zukunft können nicht nur identifiziert und studiert werden. Die Zukunft selbst kann beeinflusst werden (Berger et al. 2008).

Bei der Szenario-basierten Vorausschau lassen sich zwei wesentliche Stoßrichtungen unterscheiden: die Szenario-Technik und das Scenario Planning. Bei der Szenario-Technik werden induktiv Szenarien erarbeitet. D. h., dass systematisch konsistente Kombinationen von alternativen Entwicklungsmöglichkeiten von Schlüsselfaktoren gebildet werden. Im Gegensatz dazu erfolgt die Szenario-Erstellung bei dem im angloamerikanischen Raum populären Scenario Planning deduktiv. D. h., es wird mithilfe eines vorab definierten Rahmens eine festgelegte Anzahl an Szenarien erarbeitet (Götze 1993; Fink und Siebe 2006; Schühly et al. 2020).

Anders als die induktive Szenario-Technik eignet sich das deduktive Scenario Planning nur bei thematisch eng abgegrenzten Fragestellungen. Dies liegt in erster Linie daran, dass der Rahmen für die Szenarien eher unsystematisch und unter Berücksichtigung weniger Faktoren festgelegt wird. Auf diese Weise kann es passieren, dass Szenarien, die nicht bereits absehbar oder subjektiv erwartbar sind, von Beginn an implizit ausgeschlossen werden (Schnaars 1987). Folglich wird im weiteren Verlauf dieses Beitrags ausschließlich die Szenario-Technik fokussiert.

In der Literatur haben bereits einige Autorinnen und Autoren verschiedene Ansätze der Szenario-Technik miteinander verglichen und zusammenfassende Darstellungen erarbeitet (Huss und Honton 1987; Geschka und Hammer 1990; Götze 1993; Stelzer et al. 2015). Jedoch wird hierbei nicht immer klar zwischen Ansätzen für das Scenario Planning und der Szenario-Technik differenziert. Daher werden in diesem Beitrag im Rahmen einer Metaanalyse ausschließlich Ansätze der SzenarioTechnik gegenübergestellt, um konstituierende und optionale Phasen und Schritte zu identifizieren. Abb. 2 zeigt das Ergebnis dieser Metaanalyse. In den Spalten sind einerseits generalisierte bzw. zusammenfassende Vorgehensweisen aufgeführt (vgl. hierzu die Einträge [1] bis [4] in den Spalten). Andererseits werden auch eigenständige, praxisrelevante Vorgehensweisen untersucht (vgl. hierzu die Einträge [5] bis [17] in den Spalten). Mithilfe der Metaanalyse lassen sich acht konstituierende Schritte und ein optionaler Schritt für die Szenario-Technik identifizieren, die sich wiederum drei übergeordneten Phasen zuordnen lassen: Szenario-Vorbereitung, Szenario-Erstellung, Szenario-Transfer.

Szenario-Vorbereitung Vor Beginn eines Szenario-Projekts gilt es, den Untersuchungsgegenstand zu definieren und zu analysieren (Schritt 1). Dies umfasst neben der Festlegung der Projektzielsetzung und der -organisation die Definition und Analyse des Untersuchungsgegenstands bzw. Gestaltungsfelds selbst sowie die Definition des Szenariofelds, in welches der Untersuchungsgegenstand eingebettet ist (Huss und Honton 1987; Götze 1993; Gausemeier et al. 2019). 


\begin{tabular}{|c|c|c|c|c|c|c|c|c|c|c|c|c|c|c|c|c|c|c|}
\hline \multirow[b]{2}{*}{ Phase } & \multirow[b]{2}{*}{ Schritt } & \multicolumn{17}{|c|}{ Ansätze der Szenario-Technik } \\
\hline & & 1 & 2 & 3 & 4 & 5 & 6 & 7 & 8 & 9 & 10 & 11 & 12 & 13 & 14 & 15 & 16 & 17 \\
\hline 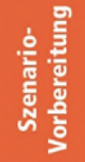 & $\begin{array}{l}\text { 1) Definition und } \\
\text { Analyse des Unter- } \\
\text { suchungsgegen- } \\
\text { stands }\end{array}$ & $\mathrm{x}$ & $x$ & $x$ & $\mathrm{x}$ & $x$ & $x$ & $x$ & $x$ & $x$ & $\mathrm{x}$ & $\mathrm{x}$ & $x$ & $x$ & $x$ & $x$ & $x$ & $\mathrm{x}$ \\
\hline \multirow{5}{*}{ 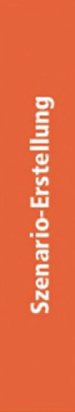 } & $\begin{array}{l}\text { 2) Identifikation von } \\
\text { Schlüsselfaktoren }\end{array}$ & $\mathrm{x}$ & $x$ & $x$ & $x$ & $x$ & $x$ & $x$ & $x$ & $x$ & $x$ & $x$ & $x$ & $x$ & $x$ & $x$ & $x$ & $x$ \\
\hline & $\begin{array}{l}\text { 3) Projektions- } \\
\text { Entwicklung }\end{array}$ & $\mathrm{x}$ & $x$ & $x$ & $x$ & $x$ & $x$ & $x$ & $x$ & $x$ & $x$ & $x$ & $x$ & $x$ & $x$ & $x$ & $x$ & $\mathrm{x}$ \\
\hline & $\begin{array}{l}\text { 4) Ermittlung von } \\
\text { Projektionsbündeln } \\
\text { und Szenarien }\end{array}$ & $\mathrm{x}$ & $x$ & $x$ & $x$ & $x$ & $x$ & $x$ & $x$ & $x$ & $x$ & $x$ & $\mathrm{x}$ & & $x$ & $x$ & $x$ & $\mathrm{x}$ \\
\hline & $\begin{array}{l}\text { 5) Sensitivitäts- } \\
\text { analyse (optional) }\end{array}$ & $\mathrm{x}$ & $x$ & & & & & & $x$ & $\mathrm{x}$ & & $x$ & & & & & & \\
\hline & $\begin{array}{l}\text { 6) Ausarbeitung } \\
\text { der Szenarien }\end{array}$ & $x$ & $x$ & $x$ & $\mathrm{x}$ & $x$ & $x$ & & $x$ & $x$ & $x$ & $x$ & & $x$ & $x$ & $x$ & $x$ & $\mathrm{x}$ \\
\hline \multirow{3}{*}{ 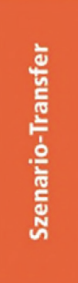 } & $\begin{array}{l}\text { 7) Auswirkungs- } \\
\text { analyse }\end{array}$ & $x$ & $x$ & $x$ & $x$ & $x$ & $x$ & & $x$ & $x$ & $x$ & $x$ & & & $x$ & $x$ & $x$ & $x$ \\
\hline & $\begin{array}{l}\text { 8) Ableitung von } \\
\text { strategischen Stoß- } \\
\text { richtungen und } \\
\text { Maßnahmen }\end{array}$ & & & $x$ & $\mathrm{x}$ & & $x$ & $x$ & & & $x$ & $\mathrm{x}$ & & & $x$ & $x$ & $\mathrm{x}$ & $\mathrm{x}$ \\
\hline & $\begin{array}{l}\text { 9) Szenario } \\
\text { Controlling }\end{array}$ & & & & & & $x$ & & & & & & & & $x$ & & & \\
\hline
\end{tabular}

\section{Legende für die Ansätze der Szenario-Technik}

1 Huss und Honton 1987

2 Götze 1993

3 Kosow et al. 2008

4 Stelzer et al. 2015

5 Oberkampf 1976

6 Segner 1976

7 Linnemann und Kennell 1977

8 Gomez und Escher 1980

9 Battelle Institut Genf in (Gomez und Escher 1980)

10 Battelle Institut Frankfurt in (Geschka und Reibnitz 1987)

11 Battelle Columbus Division in (Huss und Honton 1987)

12 Center for Future Research in (Huss und Honton 1987)

13 Godet 1987

14 Reibnitz 1982

15 Heinecke und Schwager 1995

16 Geschka 2006

17 Gausemeier et al. 2019

Abb. 2. Metaanalyse zur Szenario-Technik 
Szenario-Erstellung Die Szenario-Erstellung beginnt mit der Identifikation von Schlüsselfaktoren (Schritt 2). Dabei wird das vorab definierte Szenariofeld in Einflussbereiche strukturiert und mithilfe von Einflussfaktoren beschrieben. Die wichtigsten Einflussfaktoren, die Schlüsselfaktoren, werden anhand ihrer Vernetzung und Relevanz ausgewählt. Im Rahmen der Projektions-Entwicklung erfolgt der eigentliche Blick in die Zukunft (Schritt 3). Für jeden Schlüsselfaktor werden alternative Entwicklungsmöglichkeiten, sogenannte Zukunftsprojektionen, erarbeitet. Diese Zukunftsprojektionen werden zu konsistenten Projektionsbündeln und Szenarien verknüpft (Schritt 4). Dies kann entweder mithilfe einer intuitiven Bewertung, einer Cross Impact-Analyse oder einer paarweisen Konsistenzbewertung der Zukunftsprojektionen mit anschließender Clusteranalyse erfolgen. Anschließend lässt sich eine Sensitivitätsanalyse für die erarbeiteten Szenarien durchführen (Schritt 5). Es können sowohl Daten als auch bei der Szenario-Bildung getroffene Annahmen variiert und deren Auswirkungen analysiert werden. Dieser Schritt ist optional, weil er nur einen Bestandteil weniger Ansätze zur Szenario-Technik darstellt. Die SzenarioErstellung schließt mit der zielgruppengerechten Ausarbeitung und Aufbereitung der Szenarien (Schritt 6) (Götze 1993; Huss und Honton 1987; Gausemeier et al. 2019).

Szenario-Transfer Das Vorgehen der Auswirkungsanalyse hängt von der primären Funktion der Szenarien, in der Regel Entscheidungsunterstützung oder Orientierungswissen in unsicheren Umfeldern, ab (Schritt 7). Grundsätzlich werden zunächst die Auswirkungsstärke und die Eintrittswahrscheinlichkeiten der Szenarien evaluiert, bevor die Chancen und Gefahren für das Gestaltungsfeld systematisch analysiert werden. In diesem Zuge kann auch die Sensitivität der Szenarien in Hinblick auf mögliche Strukturbrüche, die nicht in den Szenarien berücksichtigt werden, untersucht werden. Darauf aufbauend werden strategische Stoßrichtungen und Maßnahmen für das Unternehmen abgeleitet (Schritt 8). Abschließend steht das Szenario Controlling an (Schritt 9). Dieses wird zwar nur in wenigen Ansätzen explizit als Bestandteil der Szenario-Technik beschrieben. In vielen Ansätzen wird das Szenario Controlling aber zumindest angerissen und sollte daher ebenfalls zu den konstituierenden Schritten gezählt werden. Inhaltlich geht es einerseits um das Monitoring der erarbeiteten Szenarien. D. h., es wird überprüft, welche Szenarien im Zeitverlauf wahrscheinlicher werden und folglich an Bedeutung gewinnen. Andererseits gehört auch die Analyse der Validität und Aussagekraft der Szenarien zum Szenario Controlling. Hierbei wird entschieden, ob und in welchem Umfang die Szenarien aktualisiert und überarbeitet werden müssen (Fink und Siebe 2006; Schühly et al. 2020; Götze 1993).

In der Praxis ist der Einsatz der Szenario-Technik mit einigen Herausforderungen verbunden. Diese führen gemeinsam mit den immer komplexer werdenden Unternehmensumfeldern zu einem Rückgang der Nutzungszahlen (vgl. Abschn. 1). Für die Erarbeitung eines Lösungsansatzes für eine digital souveräne Szenario-Technik mit digitalen Technologien wird die Design Science Research Methodology genutzt (Blessing und Chakrabarti 2009). Diese Forschungsmethode gliedert sich in vier 
Phasen: Klärung der Forschungsfragen, deskriptive Studie I, präskriptive Studie, deskriptive Studie II.

Dieser Beitrag beschäftigt sich ausschließlich mit der ersten Phase. Ziel sind konkrete Handlungsfelder zur Strukturierung des Forschungsfelds, welche den Ausgangspunkt für die Erarbeitung des Lösungsansatzes darstellen (Phase zwei bis vier). Zunächst gilt es dabei, Herausforderungen bei der Anwendung der Szenario-Technik (vgl. Abschn. 3) und Potenziale digitaler Technologien für die Szenario-Technik zu identifizieren (vgl. Abschn. 4). Die explorative Identifikation von Herausforderungen und Potenzialen wurde im Rahmen eines Expertenworkshops mit je drei Experten aus den Bereichen Szenario-Technik und digitale Technologien durchgeführt. Basierend auf diesen Erkenntnissen und einer Betrachtung der relevanten Literatur werden abschließend drei Handlungsfelder für die Erarbeitung des Lösungsansatzes formuliert (vgl. Abschn. 5).

\section{Herausforderungen der Szenario-Technik}

Bevor Herausforderungen bei der Anwendung der Szenario-Technik identifiziert werden können, gilt es, ein allgemeines Verständnis für die Szenario-Technik aufzubauen. Hierzu wird ein Prozessmodell der Szenario-Technik mithilfe der Modellierungssprache OMEGA modelliert und mithilfe der Designprinzipien nach vom Brocke angereichert und ausgestaltet (Fahrwinkel 1995; vom Brocke 2007). Den Ausgangspunkt für das Prozessmodell stellen die in Abschn. 2 vorgestellten Phasen und Schritte dar. Diese werden um diejenigen Tätigkeiten ergänzt, die häufig in den analysierten Ansätzen für die Szenario-Technik genannt werden. So werden z. B. im Rahmen des Schrittes „Identifikation von Schlüsselfaktoren“ u. a. die Tätigkeiten „Einflussfaktoren bestimmen“ sowie „Einfluss- und Relevanzanalyse durchführen“ durchlaufen (vgl. Abb. 3).

Im Rahmen des Expertenworkshops konnten entlang des Prozessmodells für die Szenario-Technik mittels Brainwriting und Brainstorming insgesamt 34 Herausforderungen gesammelt werden, die sich den Phasen, Schritten oder Tätigkeiten zuordnen lassen. Die Herausforderungen können durch Entfernung von Dopplungen und der Zusammenfassung nach Ähnlichkeit zu 22 Herausforderungen zusammengefasst werden. Abb. 3 zeigt einen Ausschnitt des Prozessmodells der SzenarioTechnik mit zwei exemplarischen Herausforderungen bei der Identifikation von Schlüsselfaktoren. 
2) Identifikation von Schlüsselfaktoren

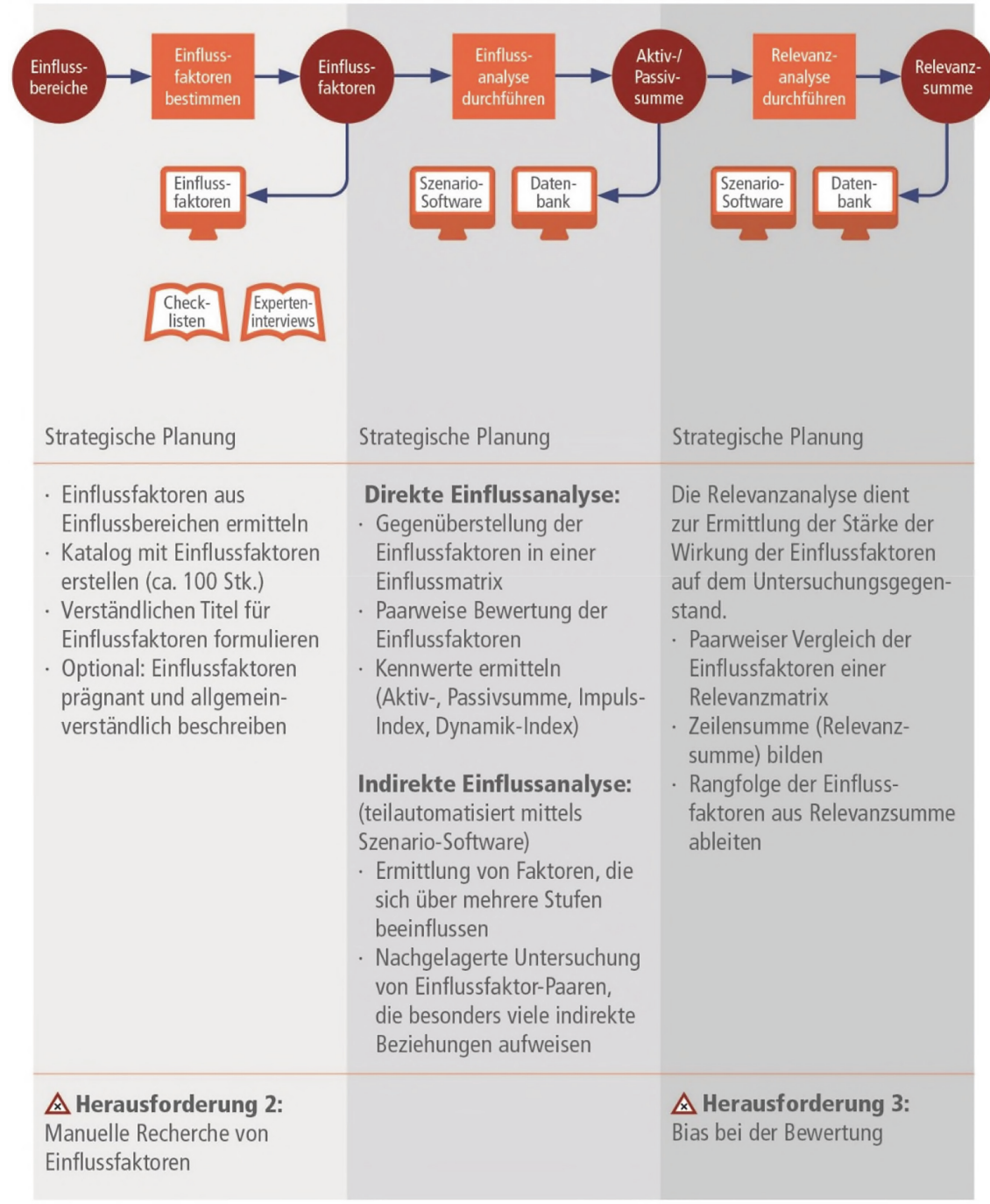

Abb. 3. Ausschnitt aus dem Prozessmodell der Szenario-Technik mit exemplarischen Herausforderungen

Zunächst einmal ist für die Bestimmung der Einflussfaktoren eine aufwendige manuelle Recherche notwendig (Herausforderung 2). Typischerweise lassen sich allgemeine, wiederkehrende Einflussfaktoren mithilfe von Checklisten identifizieren, während themenspezifische Einflussfaktoren durch Experteninterviews ermittelt werden (Gausemeier et al. 2019). Die sich anschließenden Tätigkeiten zur Einflussund Relevanzanalyse zur Ermittlung der Schlüsselfaktoren sehen sich mit der Herausforderung von Bias bei der Bewertung konfrontiert (Herausforderung 3). 
Allgemein lässt sich konstatieren, dass die Vorteile der Szenario-Technik gegenüber anderen Werkzeugen der strategischen Planung und Vorausschau um den Preis eines hohen Informationsbedarfs und damit verbundenem Datenerhebungsaufwand erkauft werden (Backhaus et al. 2018). Dabei steigt die Anzahl relevanter Informationsquellen, z. B. durch Social Media, während die Halbwertszeit von Informationen im Zuge der Digitalisierung deutlich sinkt (Belger et al. 2019).

Während die Szenario-Technik die Vielfalt und Vernetzung von Einflussfaktoren gut abbilden kann, stößt sie bedingt durch die zunehmende Dynamik der Unternehmensumfelder an ihre Grenzen. Digitale Technologien besitzen das Potenzial, die Effizienz der Szenario-Erstellung und die Evidenz der Ergebnisse zu erhöhen und damit die Erstellung von und die Arbeit mit Szenarien entscheidend zu verbessern. In der Praxis existieren bereits erste Ansätze für digitale Technologien (acatech 2020; Schühly et al. 2020; Ködding und Dumitrescu 2020)

\section{Potenziale digitaler Technologien}

Ausgehend von den identifizierten Herausforderungen der Szenario-Technik gilt es, Ideen bzw. Potenziale für digitale Technologien zur Lösung der Herausforderungen zu sammeln. Digitale Technologien werden dabei als Wissen, Kenntnisse und Fertigkeiten zur Erstellung, Verarbeitung, Übertragung und Nutzung digitaler Daten sowie Anlagen und Verfahren zur praktischen Umsetzung aufgefasst (Lipsmeier et al. 2018; Loebbecke 2006). Im Fokus stehen für die Szenario-Technik Analytics-getriebene digitale Technologien, wie z. B. virtuelle Assistenten oder Machine LearningAnwendungen, die sich im Vergleich zu weiteren digitalen Technologien insbesondere zur Steigerung der Effizienz einzelner Tätigkeiten sowie der Evidenz der Ergebnisse eignen (Berger et al. 2018). Für die Identifikation von Potenzialen für digitale Technologien wurden im Expertenworkshop Hilfsmittel zur Anregung der Kreativität der Teilnehmenden während des Brainwritings und Brainstormings genutzt. Das Periodensystem der Künstlichen Intelligenz wurde verwendet, um die Beschreibung von Tätigkeiten, die durch digitale Technologien gelöst werden sollen, sowie die Generierung erster Lösungsideen zu unterstützen (Bitkom 2018). Weiterhin wurden die Teildisziplinen künstlicher Intelligenz nach KPMG für die Ermittlung konkreter digitaler Technologien eingesetzt (Pütter 2018). Mit diesen Hilfsmitteln konnten im Expertenworkshop insgesamt 21 unterschiedliche Potenziale für digitale Technologien ermittelt werden. Abb. 4 zeigt einen Auszug der Liste der identifizierten Herausforderungen und Potenziale sowie deren Zuordnung zu den Phasen, Schritten und Tätigkeiten der Szenario-Technik. 


\begin{tabular}{|c|c|c|c|c|c|c|}
\hline \multicolumn{3}{|c|}{ Szenario-Technik } & \multicolumn{2}{|c|}{ Herausforderung } & \multicolumn{2}{|c|}{ Potential } \\
\hline Phase & Schritt & Tätigkeit & Nr. & Beschreibung & Nr. & Beschreibung \\
\hline Übergeordnet & - & - & $\mathrm{H} 1$ & $\begin{array}{l}\text { Zeitliche Dauer und } \\
\text { Aufwand von klassi- } \\
\text { schen Szenario- } \\
\text { Projekten }\end{array}$ & & $\begin{array}{l}\text { Allgemein: Effizienz- } \\
\text { steigerung durch } \\
\text { digitale Technologien }\end{array}$ \\
\hline Übergeordnet & - & - & - & - & P1 & $\begin{array}{l}\text { Virtueller Assistent zur } \\
\text { Schritt-für-Schritt-An- } \\
\text { leitung bei Szenario- } \\
\text { Projekten }\end{array}$ \\
\hline $\begin{array}{l}\text { Szenario- } \\
\text { Erstellung }\end{array}$ & $\begin{array}{l}\text { 2) Identi- } \\
\text { fikation von } \\
\text { Schlüssel- } \\
\text { faktoren }\end{array}$ & $\begin{array}{l}\text { Einfluss- } \\
\text { faktoren } \\
\text { bestimmen }\end{array}$ & $\mathrm{H} 2$ & $\begin{array}{l}\text { Manuelle Recherche } \\
\text { von Einflussfaktoren }\end{array}$ & P2 & $\begin{array}{l}\text { Natural Language } \\
\text { Processing zur } \\
\text { Identifikation, Analyse } \\
\text { und Bewertung von } \\
\text { Quellen (Output:Vor- } \\
\text { schläge für Einfluss-I } \\
\text { Schlüsselfaktoren) }\end{array}$ \\
\hline $\begin{array}{l}\text { Szenario- } \\
\text { Erstellung }\end{array}$ & $\begin{array}{l}\text { 2) Identi- } \\
\text { fikation von } \\
\text { Schlüssel- } \\
\text { faktore }\end{array}$ & $\begin{array}{l}\text { Einfluss- } \\
\text { analyse } \\
\text { durchführen; } \\
\text { Relevanz- } \\
\text { analyse } \\
\text { durchführen }\end{array}$ & H3 & $\begin{array}{l}\text { Bias bei der } \\
\text { Bewertung }\end{array}$ & P3 & $\begin{array}{l}\text { Co-Wort-Analysen zur } \\
\text { Ermittlung von Ein- } \\
\text { flüssen; Bag-of-Word- } \\
\text { Ansätze }\end{array}$ \\
\hline $\begin{array}{l}\text { Szenario- } \\
\text { Erstellung }\end{array}$ & $\begin{array}{l}\text { 2) Identi- } \\
\text { fikation von } \\
\text { Schlüssel- } \\
\text { faktoren }\end{array}$ & $\begin{array}{l}\text { Einfluss- } \\
\text { analyse } \\
\text { durchführen; } \\
\text { Relevanz- } \\
\text { analyse } \\
\text { durchführen }\end{array}$ & H3 & $\begin{array}{l}\text { Bias bei der } \\
\text { Bewertung }\end{array}$ & P4 & $\begin{array}{l}\text { Repräsentation der } \\
\text { Einflussfaktoren als } \\
\text { Ontologie, Bewertung } \\
\text { der Vernetzungen } \\
\text { durch Datenanalysen }\end{array}$ \\
\hline$\cdots$ & $\ldots$ & $\ldots$ & $\ldots$ & $\ldots$ & $\ldots$ & $\ldots$ \\
\hline $\begin{array}{l}\text { Szenario- } \\
\text { Transfer }\end{array}$ & $\begin{array}{l}\text { 10) Szenario } \\
\text { Controlling }\end{array}$ & - & $\mathrm{H} 22$ & $\begin{array}{l}\text { Sprunghafte } \\
\text { Entwicklungen, } \\
\text { Strukturbrüche }\end{array}$ & & \\
\hline
\end{tabular}

Abb. 4. Auszug aus der Liste an identifizierten Herausforderungen und Potenzialen

Anzumerken sei an dieser Stelle, dass nicht für alle identifizierten Herausforderungen Potenziale identifiziert werden konnten, und dass für manche Herausforderungen mehrere Lösungsansätze denkbar sind. So sind beispielsweise zur Lösung der dritten Herausforderung, Bias bei der Bewertung der Einflussfaktoren, sowohl ein Ansatz über Co-Wort-Analysen (Potenzial 3) als auch über eine Ontologie (Potenzial 4) oder eine Kombination beider Ansätze möglich. Abb. 5 verdeutlicht schematisch das Potenzial von digitalen Technologien für die Identifikation von Einfluss- und Schlüsselfaktoren innerhalb der Szenario-Technik (Herausforderung 2, Potenzial 2). 


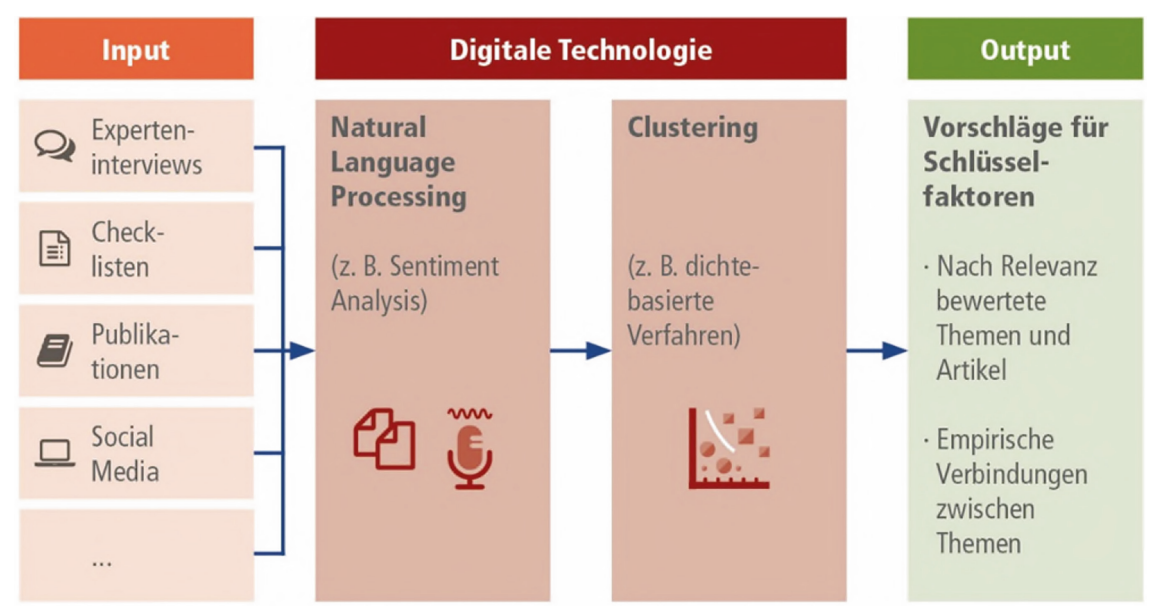

Abb. 5. Potenzial für digitale Technologien in der Szenario-Technik am Beispiel der Ermittlung von Einfluss- und Schlüsselfaktoren. (Eigene Darstellung nach Schühly et al. 2020; Sohrabi et al. 2019)

Als Inputgrößen können einerseits die für die Identifikation von Einflussfaktoren typischen Checklisten oder (transkribierte) Experteninterviews genutzt werden. Andererseits können aber auch iterativ erweiterte und verfeinerte Schlagwörter genutzt werden, um relevante Publikationen, Patente, Beiträge in Social Media etc. für den jeweiligen Anwendungskontext zu identifizieren. Im Anschluss können die Inputgrößen mithilfe von Verfahren aus dem Bereich Natural Language Processing, z. B. Sentiment Analysis, mit anschließendem Clustering, z. B. über dichtebasierte Verfahren, genutzt, analysiert und verdichtet werden. Als Ergebnis liegen nach Relevanz bewertete Themen und Artikel sowie empirisch ermittelte Vernetzungen zwischen diesen vor, aus denen sich vergleichsweise einfach Einflussfaktoren und potenzielle Schlüsselfaktoren ableiten bzw. sogar vorschlagen lassen.

In der Praxis existieren bereits erste Ansätze auf Basis von Analytics-getriebenen, digitalen Technologien, um die Anwendungsfreundlichkeit der Szenario-Technik, insbesondere aber den Datenerhebungsaufwand, zu reduzieren und die Evidenz der Ergebnisse zu steigern (z. B. Mohr 2016; Backhaus et al. 2018; Belger et al. 2019; Sohrabi et al. 2019; Schühly et al. 2020). Digitale Technologien versprechen dabei die Möglichkeit, Massendaten zu erfassen, zu analysieren und daraus Schlüsse zu ziehen (acatech 2020), (Belger et al. 2019). Insgesamt zeigt sich also, dass Analyticsgetriebene, digitale Technologien die Herausforderungen bei der Erstellung von und Arbeit mit Zukunftsszenarien adressieren und zu einem geringeren Ressourceneinsatz führen können. Dies kann wiederum Unternehmen zu einer verstärkten Nutzung der Szenario-Technik für die strategische Vorausschau befähigen. Allerdings ergeben sich durch die Integration und Nutzung von digitalen Technologien wiederum neue Herausforderungen für die Anwender, die insbesondere die digitale Souveränität betreffen. 


\section{Handlungsfelder}

Aufbauend auf den identifizierten Herausforderungen und Potenzialen für digitale Technologien lassen sich drei Handlungsfelder aufstellen, die Orientierung für die Erarbeitung des Lösungsansatzes geben und die in diesem Zuge iterativ adaptiert werden (Blessing und Chakrabarti 2009, Bortz und Döring 2006): „Integration von digitalen Technologien“, „methodische Anpassung und Weiterentwicklung der Szenario-Technik“, ,digitale Souveränität“". Nachfolgend werden die einzelnen Handlungsfelder erläutert:

\section{Handlungsfeld 1: Integration von digitalen Technologien}

Bevor digitale Technologien in die Szenario-Technik integriert werden können, müssen zunächst konkrete Use Cases für digitale Technologien identifiziert und spezifiziert werden; sowohl durch die Befragung von Anwenderunternehmen als auch die Auswertung wissenschaftlicher Publikationen. Erstens gilt es dabei, systematisch Herausforderungen bei der Anwendung der Szenario-Technik zu ermitteln. Zweitens werden Potenziale für Analytics-basierte, digitale Technologien identifiziert. Dies kann sowohl auf Grundlage der Herausforderungen aber auch losgelöst von den Herausforderungen anhand der Nutzenpotenziale digitaler Technologien erfolgen. Drittens werden die Herausforderungen und Potenziale zusammengeführt und in Form von Use Cases spezifiziert. Die Abschn. 3 und 4 liefern hierfür erste Inhalte.

Darauf aufbauend erfolgt die Identifikation von digitalen Technologien zur Realisierung der Use Cases. Im Rahmen einer strukturierten Literaturrecherche und einer Toolstudie lassen sich bestehende Ansätze und Werkzeuge systematisch ermitteln und in einer Toolbox für die Szenario-Technik strukturieren. Mithilfe dieser Übersicht wird die Bewertung und Auswahl passender digitaler Technologien erleichtert. Anschließend erfolgt die prototypische Umsetzung bzw. Adaption und Integration in die Szenario-Technik. Die Integration und Nutzung von digitalen Technologien wirken sich sowohl auf das methodische Vorgehen bei der SzenarioTechnik als auch auf die Anforderungen an die digitale Souveränität aus.

\section{Handlungsfeld 2: Methodische Anpassung und Weiterentwicklung der Szenario- Technik}

Die Metaanalyse aus Abschn. 2 zeigt die klassischen konstituierenden und optionalen Schritte der Szenario-Technik. Durch die Integration und Nutzung von digitalen Technologien entstehen jedoch viele neue Möglichkeiten für die Szenario-Technik, z. B. durch die effiziente Berücksichtigung einer Vielzahl an Datenquellen. Einerseits können Effizienz- und Evidenzsteigerungen für bestehende Schritte erzielt werden, z. B. echtzeitfähige Sensitivitätsanalysen für Strukturbrüche im Sinne einer dynamischen Szenario-Technik (Schühly et al. 2020). Andererseits können digitale Technologien gänzlich neue Schritte für die Szenario-Technik ermöglichen. Dies würde in einer grundlegenden Weiter- bzw. Neuentwicklung der Methodik resultieren. In beiden Fällen steht die Frage, welche Tätigkeiten in welchem Umfang vom Menschen und welche von digitalen Technologien ausgeführt werden, im Mittelpunkt.

\section{Handlungsfeld 3: Digitale Souveränität}

Die digitale Souveränität stellt für die beiden vorab vorgestellten Handlungsfelder ein Querschnittsthema dar. Dabei bezeichnet die digitale Souveränität die Fähigkeit 
zu selbstbestimmten Handeln und Entscheiden im digitalen Raum. Sie umfasst die Fähigkeit, digitale Technologien kompetent und zielgerichtet für die eigenen Zwecke so einzusetzen, dass die eigene Handlungs- und Entscheidungsfähigkeit mindestens erhalten bleibt (Bitkom 2015; Bogenstahl und Zinke 2017; Bitkom 2019; Institut für Innovation und Technik 2019; Hartmann 2020). Einerseits gilt es zu untersuchen, inwiefern die durch digitale Technologien unterstützte Szenario-Technik einen Beitrag zur digitalen Souveränität von Unternehmen leisten kann. Andererseits gilt es, Unternehmen unabhängig von ihrem Digitalisierungsgrad zu einer eigenständigen, digital souveränen Nutzung der Szenario-Technik mit digitalen Technologien zu befähigen. Hierzu zählt insbesondere der Aufbau der dafür erforderlichen Kompetenzen (u. a. Data Literacy) und die anwenderfreundliche Gestaltung der Interaktion von Anwendern und Anwenderinnen sowie den digitalen Technologien. Gleichzeitig müssen auch Herausforderungen adressiert werden, die erst durch die Integration und Nutzung von digitalen Technologien entstehen. Für eine hohe Akzeptanz und ein hohes Vertrauen müssen die eingesetzten digitalen Technologien vor allem die Anforderungen der Robustheit und Erklärbarkeit erfüllen; sowohl was die Ergebnisse als auch deren Zustandekommen angeht.

\section{Zusammenfassung}

Durch den Megatrend Digitalisierung eröffnen sich für Industrieunternehmen zahlreiche neue Möglichkeiten. Gleichzeitig werden Unternehmen allerdings durch immer dynamischere Veränderungen in immer komplexer werdenden Umfeldern, rasant steigende Datenmengen und die sinkende Halbwertszeit von Informationen vor große Herausforderungen bei der strategischen Vorausschau mit der Szenario-Technik gestellt. Digitale Technologien stellen einen Erfolg versprechenden Ansatz dar, die Erstellung von und Arbeit mit Szenarien signifikant zu verbessern; vor allem durch die Reduktion des Datenerhebungsaufwands und des Ressourceneinsatzes sowie die Steigerung der Evidenz der Ergebnisse.

Im vorliegenden Beitrag wurde die Szenario-Technik, Herausforderungen bei ihrer Anwendung und Potenziale zu deren Lösung durch Analytics-getriebene, digitale Technologien analysiert. Aus den Herausforderungen und Potenzialen lassen sich drei Handlungsfelder für die Erarbeitung eines Lösungsansatzes für eine Szenario-Technik mit digitalen Technologien ermitteln: „Integration von digitalen Technologien“, „methodische Anpassung und Weiterentwicklung der Szenario-Technik“ sowie „digitale Souveränität“".

\section{Literatur}

acatech (Hrsg.): Künstliche Intelligenz in der Industrie. acatech HORIZONTE, München (2020)

Backhaus, K., König, U., Mohr, S., Paulsen, T.: Szenarioanalyse - Verbesserungen aus der Münsteraner Effizienz-Werkstatt. In: Gausemeier, J., Bauer, W., Dumitrescu, R. (Hrsg.) Vorausschau und Technologieplanung. Symposium für Vorausschau und Technologieplanung Bd. 14, S. 261-287. Berlin, 8.-9. November 2018. Universität Paderborn Heinz Nixdorf Institut, Paderborn (2018) 
Bain \& Company, Inc. (Hrsg.): Management tools. Scenario and contingency planning. https:// www.bain.com/insights/management-tools-scenario-and-contingency-planning/ (2018). Zugegriffen: 18. Juli 2021

Barton, D., Manyika, J., Koller, T., Palter, R., Godsall, J., Zoffer, J.: Measuring the Economic Impact of Short-termism. https://www.mckinsey.com/ /media/mckinsey/featured $\% 20$ insights/long\%20term\%20capitalism/where $\% 20$ companies $\% 20$ with $\% 20 \mathrm{a} \% 201$ ong $\% 20$ term\%20view\%20outperform\%20their\%20peers/mgi-measuring-the-economic-impact-ofshort-termism.ashx (2018). Zugegriffen: 18. Juli 2021

Becker, P.: Corporate Foresight in Europe: A First Overview. European Commission. Brüssel. Working Paper European Commission (2002)

Belger, A., Blum, R., Zimmermann, R.: Maschinelle Analyse und Modellierung von Texten zum Monitoring von Markt- und Technologieumgebungen. In: Gausemeier, J., Bauer, W., Dumitrescu, R. (Hrsg.) Vorausschau und Technologieplanung. Symposium für Vorausschau und Technologieplanung Bd. 15, S. 499-512. Berlin, 21.-22. November 2019. Universität Paderborn Heinz Nixdorf Institut, Paderborn (2019)

Berger, G., Bourbon Busset, J. de, Massé, P.: De la prospective. Textes fondamentaux de la prospective française, 1955-1966. (Hrsg.) von Philippe Durance. 2. Aufl. L'Harmattan, Paris (2008)

Berger, S., Denner, M.-S., Röglinger, M.: The nature of digital technologies. Development of a multi-layer taxonomy. In: Proceedings of the $26^{\text {th }}$ European Conference on Information Systems (ECIS) S. 1-18, United Kingdom, Portsmouth, 23-28 Juni 2018 (2018)

Bitkom (Hrsg.): Digitale Souveränität. Positionsbestimmung und erste Handlungsempfehlungen für Europa. Bitkom Bundesverband Informationswirtschaft, Telekommunikation und neue Medien e. V. https://www.bitkom.org/sites/default/files/file/ import/BITKOM-Position-Digitale-Souveraenitaet.pdf (2015). Zugegriffen: 23. Juli 2021

Bitkom (Hrsg.): Digitalisierung gestalten mit dem Periodensystem der Künstlichen Intelligenz. Ein Navigationssystem für Entscheider. Bundesverband Informationswirtschaft, Telekommunikation und neue Medien e. V. (2018)

Bitkom (Hrsg.): Digitale Souveränität: Anforderungen an Technologie- und Kompetenzfelder mit Schlüsselfunktion. Bitkom Bundesverband Informationswirtschaft, Telekommunikation und neue Medien e. V. https://www.bitkom.org/sites/default/files/2020-01/200116_stellungnahme_digitale-souveranitat.pdf (2019). Zugegriffen: 23. Juli 2021

Blessing, L.T.M., Chakrabarti, A.: DRM, a design research methodology. Springer, London (2009)

Bogenstahl, C., Zinke, G.: Digitale Souveränität - ein mehrdimensionales Handlungskonzept für die deutsche Wirtschaft. In: Wittpahl, V. (Hrsg.) Digitale Souveränität, S. 65-82. Springer, Berlin (2017)

Bortz, J., Döring, N.: Forschungsmethoden und Evaluation, 4. Aufl. Springer, Berlin (2006)

Dumitrescu, R., Gausemeier, J.: Innovationen im Zeitalter der Digitalisierung. Industrie 4.0 Management (2). (2018)

Echterhoff, B., Gausemeier, J., Koldewey, C., Mittag, T., Schneider, M., Seif, H.: Geschäftsmodelle für die Industrie 4.0. In: Jung, H.H., Kraft, P. (Hrsg.) Digital vernetzt. Transformation der Wertschöpfung S. 35-56. Hanser, München (2016)

Fahrwinkel, U.: Methoden zur Modellierung und Analyse von Geschäftsprozessen zur Unterstützung des Business Process Reengineering. Dissertation. Paderborn, Fakultät für Maschinenbau, Universität Paderborn, HNI-Verlagsschriftenreihe, Band 1, Paderborn. (1995)

Fink, A., Siebe, A.: Handbuch Zukunftsmanagement. Werkzeuge der strategischen Planung und Früherkennung. Campus, Frankfurt a. M. (2006) 
Gausemeier, J., Dumitrescu, R., Echterfeld, J., Pfänder, T., Steffen, D., Thielemann, F.: Innovationen für die Märkte von morgen. Strategische Planung von Produkten, Dienstleistungen und Geschäftsmodellen. Hanser, München (2019)

Gausemeier, J., Plass, C.: Zukunftsorientierte Unternehmensgestaltung. Strategien, Geschäftsprozesse und IT-Systeme für die Produktion von morgen, 2. Aufl. Hanser, München (2014)

Geschka, H.: Szenariotechnik als Instrument der Frühaufklärung. In: Gassmann, O., Kobe, C. (Hrsg.) Management von Innovation und Risiko, S. 357-372. Heidelberg, Springer (2006)

Geschka, H., Hammer, R.: Die Szenario-Technik in der strategischen Unternehmensplanung. In: Hahn, D., Taylor, B. (Hrsg.) Strategische Unternehmungsplanung / Strategische Unternehmungsführung. Stand und Entwicklungstendenzen S. 311-336. Physica-Verlag HD, Heidelberg (1990)

Geschka, H., Reibnitz, U. Von: Die Szenario-Technik - ein Instrument der Zukunftsanalyse und der strategischen Planung. In: Töpfer, A., Afheldt, H., Andreae, M. (Hrsg.) Praxis der strategischen Unternehmensplanung, 2. Aufl., S. 125-170. Poller, Stuttgart (1987)

Godet, M.: Scenarios and strategic management. Butterworth, London (1987)

Gomez, P., Escher, F.: Szenarien als Planungshilfen. Management-Zeitschrift Industrielle Organisation 49(9), 416-420 (1980). https://www.alexandria.unisg.ch/id/eprint/11119

Götze, U.: Szenario-Technik in der strategischen Unternehmensplanung. Zugl.: Göttingen, Univ., Dissertation, 1990. 2. Aufl., Deutscher Universitätsverlag, Wiesbaden (1993)

Hartmann, E.A.: Digitale Souveränität in der Wirtschaft - Gegenstandsbereiche, Konzepte und Merkmale. In: Hartmann, E.A. (Hrsg.) Digitalisierung souverän gestalten, S. 1-16. Springer, Berlin (2020)

Heinecke, A., Schwager, M.: Die Szenario-Technik als Instrument der strategischen Planung. Technical University, Braunschweig (1995)

Huss, W.R., Honton, E.J.: Scenario planning-what style should you use? Long Range Plan. 20(4), 21-29 (1987). https://doi.org/10.1016/0024-6301(87)90152-X

Institut für Innovation und Technik (Hrsg.): Bekanntmachung. Promotionsbegleitende Zuschüsse (2019-2023) im Rahmen eines interdisziplinären Graduiertennetzwerks zum Themenbereich „Maschinenbau der Zukunft“. https://www.iit-berlin.de/de/aktuelles/Bekan ntmachungGraduiertennetzwerkDigitaleSouvernittinderWirtscha...pdf (2019). Zugegriffen: 12. Okt. 2019

Ködding, P., Dumitrescu, R.: Forschungsfelder für Künstliche Intelligenz in der strategischen Produktplanung. In: Hartmann, E.A. (Hrsg.) Digitalisierung souverän gestalten, S. 59-73. Springer, Berlin (2020)

Kosow, H., Gaßner, R., Erdmann, L.: Methoden der Zukunfts- und Szenarioanalyse. Überblick, Bewertung und Auswahlkriterien. IZT, Berlin (2008)

Linnemann, R.E., Kennell, J.D.: Shirt-sleeve approach to long-range plans. Harv. Bus. Rev. (März), 141-150 (1977). https://hbr.org/1977/03/shirt-sleeve-approach-to-long-range-plans

Lipsmeier, A., Bansmann, M., Roeltgen, D., Kuerpick, C.: Framework for the identification and demand-orientated classification of digital technologies. In: 2018 IEEE International Conference on Technology Management, Operations and Decisions (ICTMOD), Marrakech, Morocco, 21.-23. November 2018. IEEE, 31-36 (2018)

Loebbecke, C.: Digitalisierung. Technologien und Unternehmensstrategien. In: Scholz, C. (Hrsg.) Handbuch Medienmanagement. Mit 46 Tabellen, S. 357-373. Springer, Berlin (2006)

Mohr, S.: Decomposing consistency matrices. A methodological approach to increase efficiency and applicability of consistency analysis in scenario processes. Dissertation, Münster, Westfälischen Wilhelms-Universität Münster (2016)

Oberkampf, V.: Szenario-Technik. Darstellung der Methodik. Frankfurt (1976) 
Ogilvy, J.: Scenario planning and strategic forecasting. https://www.forbes.com/sites/ stratfor/2015/01/08/scenario-planning-and-strategic-forecasting/?sh=1327b5a5411a (2015). Zugegriffen: 7. Apr. 2021

Pütter, C.: Einsatzszenarien von KI bis 2040. https://www.cio.de/a/einsatzszenarien-von-kibis-2040,3579864 (2018). Zugegriffen: 22. Juli 2021

Reibnitz, U. Von: Szenario-Technik. Instrumente für die unternehmerische und persönliche Erfolgsplanung, 2. Aufl. Gabler Verlag, Wiesbaden (1992)

Rigby, D., Bilodeau, B.: Management tools \& trends. https://www.bain.com/contentassets/caa4 0128a49c4f34800a76eae15828e3/bain_brief-management_tools_and_trends.pdf (2018)

Rohrbeck, R., Kum, M.E.: Corporate foresight and its impact on firm performance: a longitudinal analysis. Technol. Forecast. Soc. Chang. 129, 105-116 (2018). https://doi. org/10.1016/j.techfore.2017.12.013

Rohrbeck, R., Etingue Kum, M., Jissink, T., Gordon, A.V.: Corporate foresight benchmarking report 2018. How leading firms build a superior position in markets of the future. SSRN Electron. J. (2018). https://doi.org/10.2139/ssrn.3178562

Schnaars, S.P.: How to develop and use scenarios. Long Range Plan. 20(1), 105-114 (1987). https://doi.org/10.1016/0024-6301(87)90038-0

Schühly, A., Becker, F., Klein, F.: Real time strategy: when strategic foresight meets artificial intelligence. Emerald Publishing Limited (2020)

Segner, M.: Szenario-Technik. Methodische Darstellung und kritische Analyse. Forschungsreihe Systemtechnik, Bd. 8. (1976)

Sohrabi, S., Katz, M., Hassanzadeh, O., Udrea, O., Feblowitz, M.D., Riabov, A.: IBM scenario planning advisor: plan recognition as AI planning in practice. AI Commun. 32(1), 1-13 (2019). https://doi.org/10.3233/AIC-180602

Stelzer, B., Meyer-Brötz, F., Schiebel, E., Brecht, L.: Combining the scenario technique with bibliometrics for technology foresight: The case of personalized medicine. Technol. Forecast. Soc. Chang. 98, 137-156 (2015). https://doi.org/10.1016/j.techfore.2015.06.008 vom Brocke, J.: Design principles for reference modeling. In: Fettke, P., Loos, P. (Hrsg.) Reference modeling for business systems analysis, S. 47-76. IGI Global (2007)

Open Access Dieses Kapitel wird unter der Creative Commons Namensnennung 4.0 International Lizenz (http://creativecommons.org/licenses/by/4.0/deed.de) veröffentlicht, welche die Nutzung, Vervielfältigung, Bearbeitung, Verbreitung und Wiedergabe in jeglichem Medium und Format erlaubt, sofern Sie den/die ursprünglichen Autor(en) und die Quelle ordnungsgemäß nennen, einen Link zur Creative Commons Lizenz beifügen und angeben, ob Änderungen vorgenommen wurden.

Die in diesem Kapitel enthaltenen Bilder und sonstiges Drittmaterial unterliegen ebenfalls der genannten Creative Commons Lizenz, sofern sich aus der Abbildungslegende nichts anderes ergibt. Sofern das betreffende Material nicht unter der genannten Creative Commons Lizenz steht und die betreffende Handlung nicht nach gesetzlichen Vorschriften erlaubt ist, ist für die oben aufgeführten Weiterverwendungen des Materials die Einwilligung des jeweiligen Rechteinhabers einzuholen.

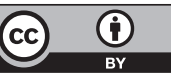

